\begin{tabular}{l|l} 
REVISTA & $\begin{array}{l}\text { Revista Educación } \\
\text { ISSN: 0379-7082 } \\
\text { ISSN: 2215-2644 } \\
\text { revedu@ gmail.com } \\
\text { Universidad de Costa Rica } \\
\text { Costa Rica }\end{array}$
\end{tabular}

\title{
Tendencias de la innovación educativa en los contextos sociales. Análisis del mapeo de literatura
}

\author{
Ramírez Ramírez, Leticia Nayeli \\ Tendencias de la innovación educativa en los contextos sociales. Análisis del mapeo de literatura \\ Revista Educación, vol. 44, núm. 1, 2020 \\ Universidad de Costa Rica, Costa Rica \\ Disponible en: http://www.redalyc.org/articulo.oa?id=44060092001 \\ DOI: https://doi.org/10.15517/revedu.v44i1.33222
}

Esta obra está bajo una Licencia Creative Commons Atribución-NoComercial-SinDerivar 3.0 Internacional. 


\title{
Tendencias de la innovación educativa en los contextos sociales. Análisis del mapeo de literatura
}

\author{
Social Contexts and Educational Innovation Trends: A Bibliographical Mapping Analysis \\ Leticia Nayeli Ramirez Ramirez \\ Universidad Panamericana, México \\ DOI: https://doi.org/10.15517/revedu.v44i1.33222 \\ Redalyc: http://www.redalyc.org/articulo.oa?id=44060092001
}

nramirez@up.edu.mx

(iD http://orcid.org/0000-0002-8113-2368

Recepción: 23 Junio 2018

Aprobación: 15 Julio 2019

\section{ReSUMEN:}

La innovación educativa aparece como un tema pendiente en las agendas de las instituciones educativas a nivel internacional. Las políticas educativas vigentes ponen de manifiesto la importancia de la innovación educativa como un elemento clave para la mejora de la calidad educativa y el desarrollo social. Desde una perspectiva sociocultural se subraya el carácter social y situado de la innovación como un proceso que acontece a través de la interacción multidisciplinaria. Se elaboró una búsqueda sistemática de la literatura en dos principales bases de datos: SCOPUS y WoS (rango temporal 2013-2017). Mediante un proceso de filtrado, con criterios de inclusión/exclusión (palabras clave, tipología de documentos, años de publicación y disciplina), y de validez (calidad y pertinencia), se delimitó la muestra total $(\mathrm{N}=43)$. Los países con mayor producción de publicaciones en la temática son Estados Unidos (5), España (4) y el Reino Unido (3). Los contextos sociales en los que se han implementado estrategias de innovación educativa son las organizaciones educativas (37), empresas (5) y hospitales (1). El tipo de innovación mayoritariamente abordado versa sobre las nuevas formas de organización de actividades (29). Se discuten los hallazgos de tres contextos sociales claves en la implementación de estrategias innovadoras, ubicando seis líneas de investigación incipientes.

Palabras Clave: Tendencia educacional, Innovación educacional, Estrategias educativas, Ambiente educacional.

\section{Abstract:}

Educational innovation is a pending item on the agendas of international educational institutions. Whereas current educational policies highlight the importance of educational innovation as a key element for improving the quality of education and promoting social development, the social aspect of innovation involves multidisciplinary interaction from a sociocultural perspective. After conducting a systematic bibliographical search of two main databases: SCOPUS and WoS (period ranging from 2013-2017), a total sample $(\mathrm{N}=43)$ was filtered with established inclusion / exclusion criteria (keywords, document type, year of publication, area of discipline) and validity (quality and relevance). The countries with the highest number of publications in this subject are the United States (5), Spain (4) and the United Kingdom (3). The social contexts in which educational innovation strategies have been executed include educational organizations (37), the corporate sector (5) and healthcare institutions (1). Innovation is addressed as it pertains to new ways of organizing activities (29). Findings involve three key social contexts for implementing innovative strategies in six emerging areas of research.

KEYWORDS: Educational trends, Educational innovation, Educational strategies, Educational environment.

\section{INTRODUCCIÓN}

En las últimas décadas, las dinámicas e interacciones humanas enmarcadas en una economía globalizada y las sociedades del conocimiento se han visto permeadas por la necesidad de dar respuesta a estándares como la eficiencia, competitividad, cobertura y producción. La innovación como concepto general ha ido cobrando importancia como un factor trascendente en el desarrollo de diversos ámbitos, incluido el educativo (OECD, 2010). Dicha relevancia radica en la potencialidad de la innovación como un vehículo para coadyuvar en la generación de cambios y mejoras sociales. 
Típicamente, la innovación ha sido una actividad circunscrita a los campos disciplinares de la tecnología, ingeniería y administración, y con aplicaciones tecnológicas en los contextos empresariales o industriales (Whitehead, 2009). Solo hasta años recientes, el uso y apropiación de la innovación se ha ido gradualmente moviendo hacia las disciplinas sociales y ha incidido en su aplicación en contextos como las escuelas, clínicas de salud, centros comunitarios y organizaciones sociales. En este sentido, la innovación educativa es entendida como un conjunto de estrategias orientadas a la implementación de una mejora significativa en un producto, servicio, proceso, organización o método relacionado con el ámbito educativo (OECD, 2015).

Desde las políticas educativas actuales, la importancia de la innovación se orienta en torno a cinco ejes: 1) las innovaciones educativas pueden mejorar los resultados del aprendizaje y la calidad de la educación, 2) es un factor de mejora en la equidad e igualdad, 3 ) tiene el potencial para estimular de manera más eficiente la entrega de los servicios educativos, 4) coadyuva en la adaptación frente al cambio acelerado en la sociedad y la economía nacional, y 5) es un elemento clave en las agendas de los programas formativos que buscan promover el desarrollo de habilidades para la innovación en los diferentes actores del sistema educativo (OECD, 2016).

Bajo este contexto se delinea a la innovación como un pilar en la promoción de las transformaciones educativas necesarias para la mejora de la calidad educativa y el desarrollo social, dichos cambios pueden ocurrir tanto a nivel macro (ej. políticas del sistema educativo, organización de la institución escolar) como a nivel micro (dinámica de la comunidad educativa, interacción estudiantes-profesores). El papel clave que juega la innovación educativa en el desarrollo y mejora de los procesos de enseñanza-aprendizaje, el comportamiento innovador de los individuos, organizaciones y economías, sin embargo, poco se ha explorado en la literatura educativa (Maritz et ál., 2014; Richmond y Tatto, 2016) sobre las características y tipología de las innovaciones educativas producidas en los diferentes contextos sociales, así como el alcance e impacto que dichas innovaciones tienen para los agentes del proceso educativo. En el mismo sentido, Ramírez (2012) subraya la importancia que tienen las estrategias de enseñanza-aprendizaje, consideradas como los planteamientos conjuntos de las directrices que determinan actuaciones concretas en cada una de las fases del proceso educativo. Estas estrategias pueden ir desde un gran marco general, como las planteadas en las políticas educativas (que, a su vez, depende de la política general del país donde se encuadran) y conducen al establecimiento detallado de una planificación educativa, hasta las estrategias micro que sigue un docente o una docente en su ambiente de aprendizaje.

De lo anterior se desprende la importancia del presente análisis del mapeo de literatura que tiene como objetivo identificar y caracterizar las tendencias investigativas producidas a nivel internacional sobre las estrategias innovadoras, con el fin de presentar un panorama general que posibilite observar nichos de acción en el área temática y los alcances de las líneas de trabajo desarrolladas.

\section{MARCo TEÓRICO}

La innovación es un concepto polisémico, en este trabajo se vislumbra desde una perspectiva sociocultural (Frane y Westlund, 2013) como un proceso social situado que no ocurre en aislado y más bien acontece a través de la interacción multidisciplinaria la cual involucra redes de colaboración entre diferentes agentes educativos, instituciones y usuarios, tales como: profesores, estudiantes, padres de familia, administrativos, empresarios y directivos. Así, los centros educativos como las universidades, colegios y bachilleratos son nodos centrales en los que ocurren procesos de innovación (OECD, 2010) y que actúan como un puente flexible para vincularse en una triple hélice: centros educativos-empresas-gobiernos. El rol central de las universidades en la empresa innovadora y su relación con los sectores empresariales ha sido ampliamente reconocido (Etzkowitz, 2012).

Si bien, la literatura sobre innovación educativa es amplia y difusa; se ha concentrado en diversos ámbitos como la innovación en la industria, comercio o tecnología, y, en diferentes momentos del proceso educativo. 
Esto se debe, por una parte, a que la innovación educativa, vista como un proceso, puede ocurrir en una gama de escenarios, contextos sociales, actores y niveles que componen la estructura educativa. Diversos autores (Aguerrondo, 1992; Hannan, English, y Silver, 1999; Ramírez y Ramírez, 2018) señalan a la innovación educativa como un proceso social situado en niveles macro y micro sociales, dicho concepto no se limita al uso de nuevas tecnologías en la organización educativa y, más bien, se identifica por propiciar un cambio a través de acciones específicas en cualquiera de los niveles sociales y los componentes de la organización. De esta manera, la innovación también se vincula con las políticas educativas y el desarrollo curricular que ocurre en los centros educativos (García, 2003) y que es validada desde dentro de los mismos por los agentes o actores que participan en ellos. A su vez, las estrategias educativas se articulan como rutas de acción específicas que el centro educativo planifica y ejecuta para generar innovaciones y evaluar el impacto que tienen estas en los agentes educativos, la comunidad y las empresas.

Siguiendo las categorías actuales propuestas por la OECD (2016), la innovación educativa podemos ubicarla en cuatro dimensiones, a saber: 1) nuevos productos o servicios, como un nuevo sílabo, libros de textos o recursos educativos; 2) nuevos procesos para entregar sus servicios como el uso de plataformas elearning; 3) nuevas formas de organizar sus actividades, ej. formas de organizar la comunicación con los estudiantes o padres de familia y 4) nuevas técnicas de marketing, por ejemplo, las estrategias de venta u ofertas para los cursos de posgrado (ver Figura 1).

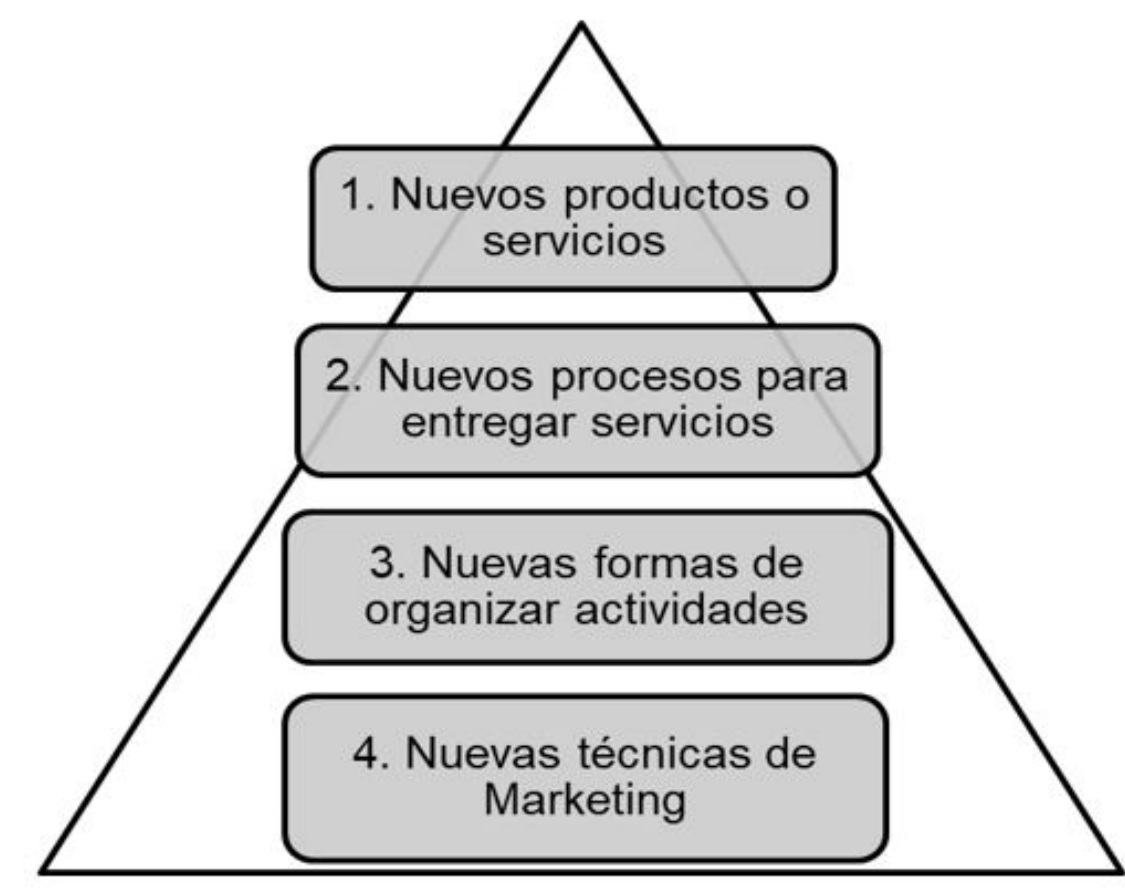

FIGURA 1

Dimensiones de la innovación educativa de acuerdo con la OECD (2016)

Fuente: Elaboración propia

\section{MÉTodo}

Se llevó a cabo un mapeo sistemático de la literatura (Petersen, Feldt, Mujtaba, y Mattsson, 2007; Tranfield, Denyer y Smart, 2003; Kitchenham et ál., 2010) el cual tiene por principal objetivo la clasificación y conducción de un análisis temático para identificar tendencias en un tema de investigación. De tal manera que se proporcione un mapa sobre las diferentes categorías generadas y su relación con el número de 
publicaciones encontradas a través de una búsqueda sistemática. La presente investigación se llevó a cabo mediante tres fases (ver Figura 2).

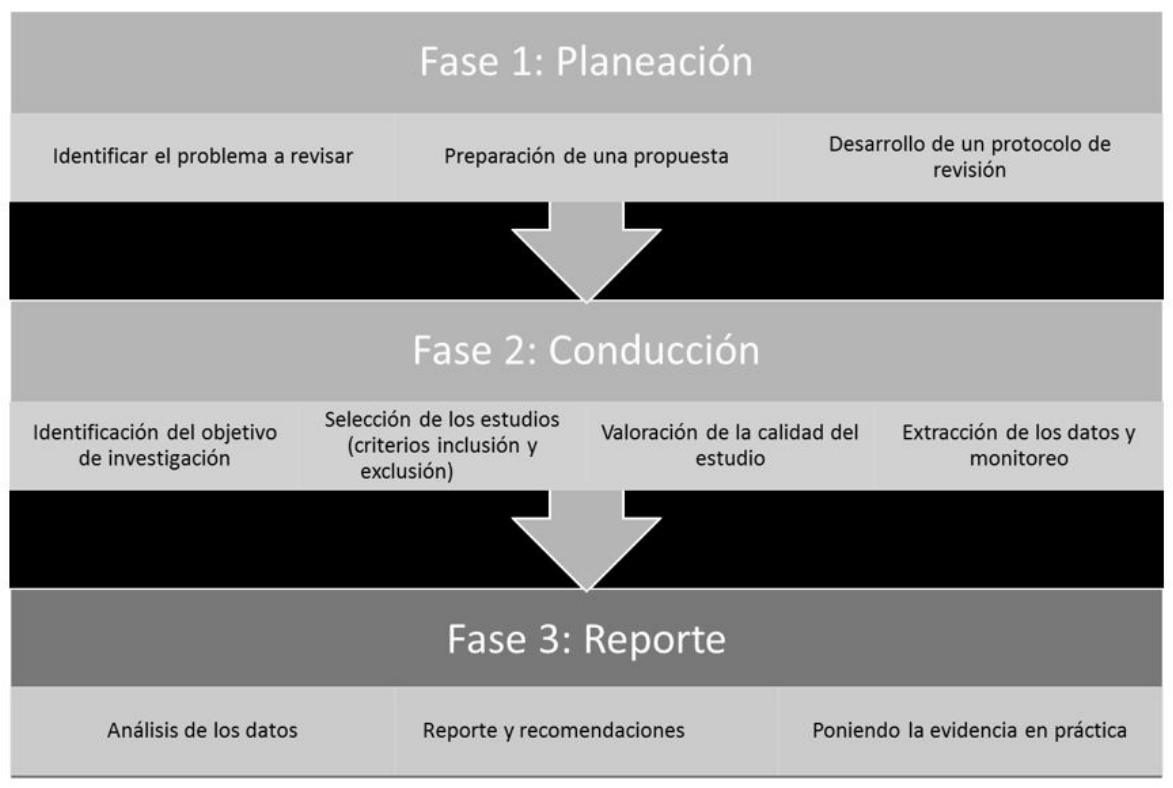

FIGURA 2

Fases del mapeo sistemático de literatura

Fuente: Modificado con información de Tranfield et ál. (2003)

\section{Fase 1. Planeación de la revisión}

En esta fase se definieron las preguntas de investigación, las palabras clave y las estrategias de búsqueda en las bases de datos. Como sugieren Petersen et ál. (2007) en el mapeo sistemático las preguntas deben alcanzar dos objetivos; identificar la cantidad y tipo de investigación producida en el área (R1, R2, R3) y las líneas, temáticas, foros en el que se han publicado dichas investigaciones (R4, R5, R6). En la Tabla 1 se presentan las preguntas de investigación que guiaron el mapeo sistemático de literatura. 
TABLA 1

Preguntas de investigación indagadas a través del mapeo sistemático de literatura

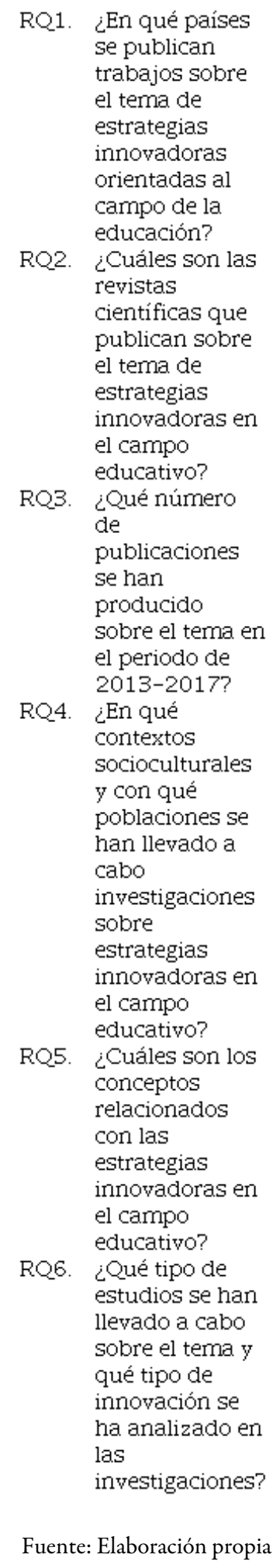

Posteriormente, se lanzó una búsqueda sistemática en dos bases de datos SCOPUS y Web of Science (WOS), la elección de dichas bases se relacionó con la calidad y prestigio de las publicaciones contenidas en 
las mismas y a la cobertura internacional que presentan. En ambas bases se mantuvieron criterios similares de búsqueda, con los siguientes criterios de inclusión:
a) Combinación de palabras clave: innovation strategy and education
b) Tipología de documentos: Artículos
c) Años de publicación: rango 2013-2017
d) Disciplina: Ciencias sociales o educación.

Se excluyeron las publicaciones que: a) correspondieran al tipo de documento: conferencias, reseñas de libros, capítulos de libro, b) rango de años antes del 2013 y c) que pertenecieran a otras áreas disciplinares que no fueran las ciencias sociales o educación. En la primera fase se obtuvo un total de 408 artículos.

Asimismo, se procedió a efectuar un vaciado de los datos, en el cual se ubicaron las siguientes dimensiones: sumatoria, palabra clave de búsqueda, base de datos, referencia bibliográfica, autores, título de la publicación, resumen, año, país de la afiliación institucional del autor principal y nombre de la revista.

\section{FASE 2. Conducción de LA REVISIÓN}

Una vez recolectados y vaciados los datos de ambas bases de datos (SCOPUS y WOS), se procedió a ejecutar la segunda selección y filtrado de publicaciones (Ver Tabla 2). En este proceso se eliminaron las publicaciones que cumplieran con los siguientes criterios de exclusión: 1) todas aquellas revistas que en sus alcances y enfoques no aceptaran temas de educación y tuvieran un énfasis hacia la economía, geografía, leyes, turismo, administración o ingeniería (como, por ejemplo: Economic Development Quarterly, International Journal of Heritage Studies, Public Organization Review, European Planning Studies, Tourism Management, Business Strategy and the Environment), y 2) todas aquellas publicaciones duplicadas en las bases de datos. En total se excluyeron un total de 268 artículos.

Posteriormente, se aplicó un último filtro que consistió en leer títulos, resúmenes de las publicaciones y, en algunos casos, el texto completo para seleccionar solo aquellos que cumplieran con los siguientes criterios de inclusión: a) que en el título hicieran referencia al campo disciplinar de la educación y, b) que dentro del contenido de los resúmenes o texto completo abordaran algún concepto relacionado con la innovación educativa.

En el proceso de selección final de la muestra se empleó una lista de evaluación checklist de la calidad y pertinencia de las investigaciones (Ver Apéndice A) con base en cuatro preguntas codificadas en una escala del 0 al 1, y revisadas por dos investigadores independientes (Kitchenham et ál., 2010). La selección final de la muestra $(\mathrm{N}=43)$ aparece numerada con las referencias completas en el Apéndice B. 
TABLA 2

Proceso de filtración realizado a través de las fases del mapeo sistemático de literatura

\begin{tabular}{|c|c|c|c|c|}
\hline \multicolumn{2}{|c|}{ FASE 1} & \multicolumn{2}{|c|}{ FASE 2} & \multirow{3}{*}{$\begin{array}{l}\text { Seleccionados } \\
\text { por } \\
\text { corresponder } \\
\text { al campo de } \\
\text { la } \\
\text { innovación } \\
\text { educativa }\end{array}$} \\
\hline \multirow[t]{3}{*}{$\begin{array}{l}\text { Palabras } \\
\text { clave }\end{array}$} & $\begin{array}{l}\text { Criterios de } \\
\text { búsqueda }\end{array}$ & \multirow{2}{*}{\multicolumn{2}{|c|}{$\begin{array}{l}\text { Eliminados: } \quad \text { Duplicados } \\
\text { Revistas de otros campos disciplinarios }\end{array}$}} & \\
\hline & $\begin{array}{l}\text { Articles, year } \\
2013-2017, \\
\text { Social } \\
\text { sciences or } \\
\text { Education }\end{array}$ & & & \\
\hline & $\begin{array}{l}\text { SCOPUS } \\
\text { WOS }\end{array}$ & SCOPUS WOS & & \\
\hline $\begin{array}{l}\text { \#innovation } \\
\text { Strategy } \\
\text { AND } \\
\text { education }\end{array}$ & $351 \quad 10$ & 259 & 2 & 43 \\
\hline Totales & 361 & 93 & & 43 \\
\hline
\end{tabular}

Fuente: Elaboración propia

\section{Resultados}

\section{Investigaciones sobre Estrategias Innovadoras en el Ámbito Educativo}

En la caracterización de las investigaciones recabadas sobre la temática de Estrategias Innovadoras, se encontró que de la muestra total identificada $(\mathrm{N}=43)$, el país que presenta una mayor frecuencia de publicación es Estados Unidos (5), seguido de España (4), Reino Unido (3), China (3), Australia (3), África (2), Dinamarca (2), Malasia (2), Taiwan (2), Eslovenia (2), y Turquía (2). Por otra parte, la frecuencia de publicación más baja (1) se presenta en: Sudáfrica, Alemania, Canadá, Chile, Finlandia, Holanda, Hong Kong, Malta, Noruega, Polonia, Suecia, Suiza, Taiwan y Tunisia. En la Figura 3, se presenta el mapa graficado en Tableau con la ubicación geográfica de los países en los que se publica la temática y la frecuencia representada en el tamaño de los círculos que ubican en el mapa a dichos países. 


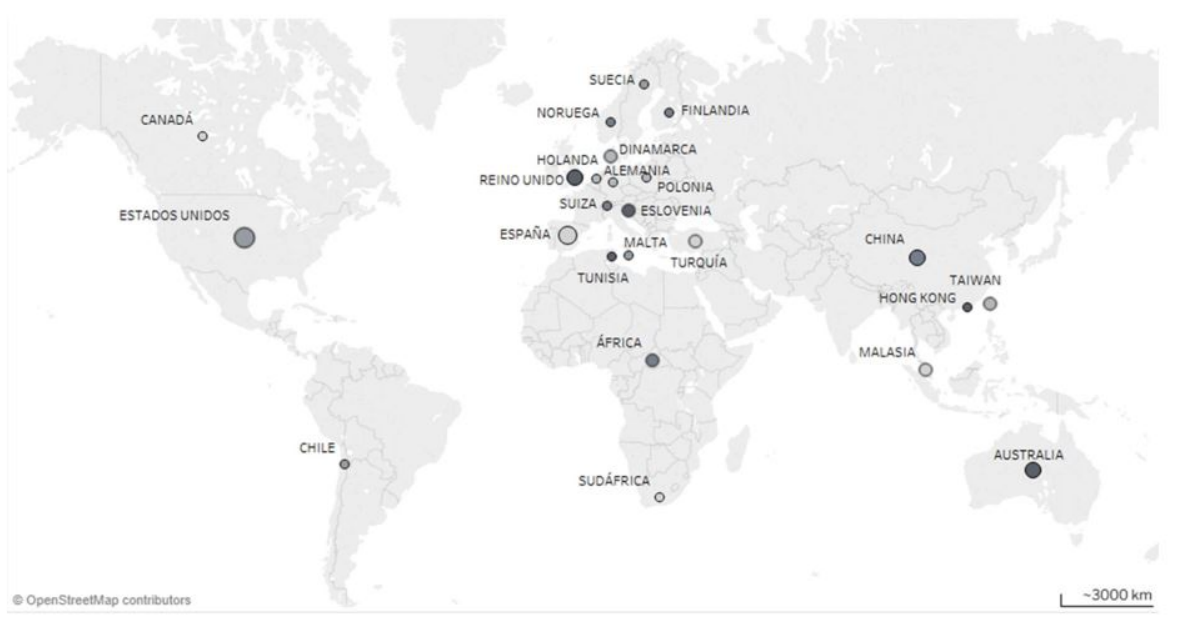

FIGURA 3

Ubicación geográfica y frecuencia de publicación sobre la temática de investigación Fuente: Elaboración propia

Por otra parte, se identificaron 38 revistas que publican sobre la temática, de las cuales cinco presentan una frecuencia de publicación alto: Journal of Vocational Behavior (2\%), Journal of Educational Media and Library Science (2\%), International Journal of Engineering Education (2\%), European Journal of Education (2\%), y Anthropologist (2\%). Asimismo, el año en el que se concentra una mayor publicación de la temática es en 2016, con 14 publicaciones. Si bien, se puede observar una fuerte tendencia a desarrollar investigación sobre estrategias innovadoras en el ámbito educativo, aún se mantiene muy incipiente la producción en esta área en los últimos cinco años; pues en el año 2013 solo se produjeron 9 publicaciones, en 2014 (7), en 2015 (9) y en el año en curso se llevan registradas un total de 7 publicaciones. En la Figura 4, se muestran los datos completos de las revistas científicas y la distribución de frecuencias de publicación por año.

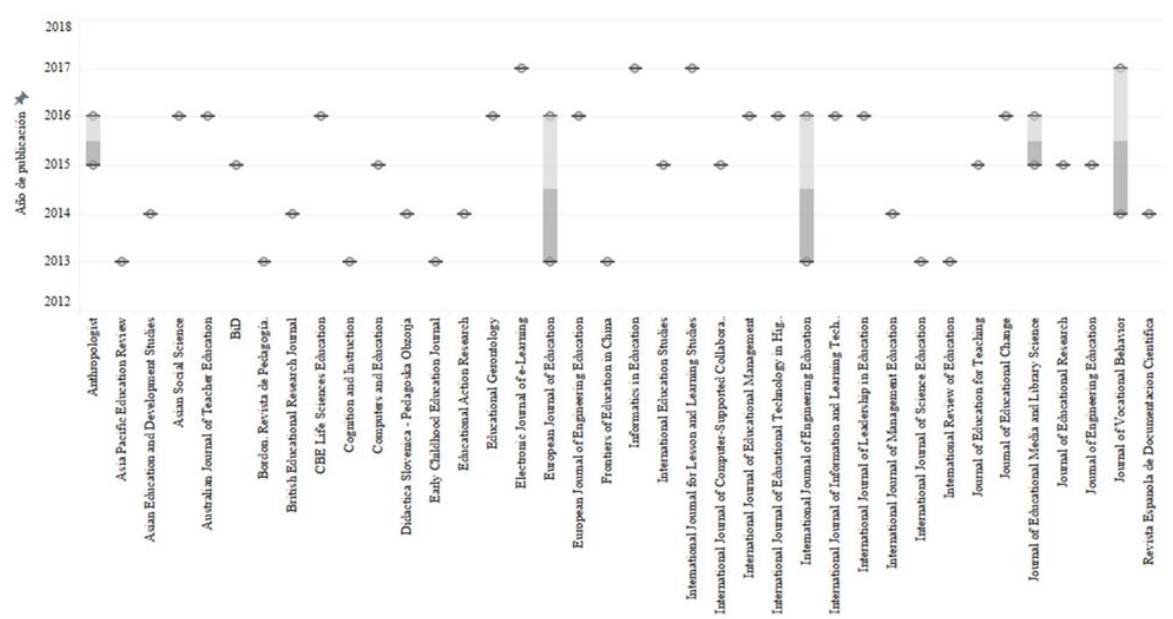

FIGURA 4

Distribución de la frecuencia de publicación en la temática por año y revista científica Fuente: Elaboración propia 


\section{Contextos sociales de las estrategias innovadoras: Organizaciones educativas, Empresas y Hospitales}

Con base en las investigaciones identificadas, emergieron tres contextos sociales en los que se puede situar la producción sobre estrategias innovadoras. Estos contextos son mayoritariamente las organizaciones educativas, 37 de los artículos se situaron en este contexto, empresas (5) y hospitales (1). Las poblaciones objetivo de las investigaciones son diversas; estudiantes (14), profesores (13), administrativos (7), trabajadores (3), bibliotecarios (2) e investigadores (1). La relación entre contextos sociales y poblaciones ubicadas en las investigaciones analizadas, se muestra en la Figura 5.

Asimismo, las investigaciones que se sitúan en el contexto de las organizaciones educativas se han interesado mayoritariamente por el nivel educativo universitario (ver las investigaciones: A2, A4, A6, A7, A16, A17, A19, A20, A22, A25, A26, A29, A30, A32, A37, A39, A43), seguido de la educación básica (ej. A12, A14, A36, A9), secundaria (ej. A13, A31) y preescolar (ver las investigaciones: A1 y A9). Por otra parte, los contextos empresariales se documentan en las investigaciones: A10, A21, A23, A34, A35. Y la única investigación situada en el contexto de hospitales se ejemplifica en la publicación A42.

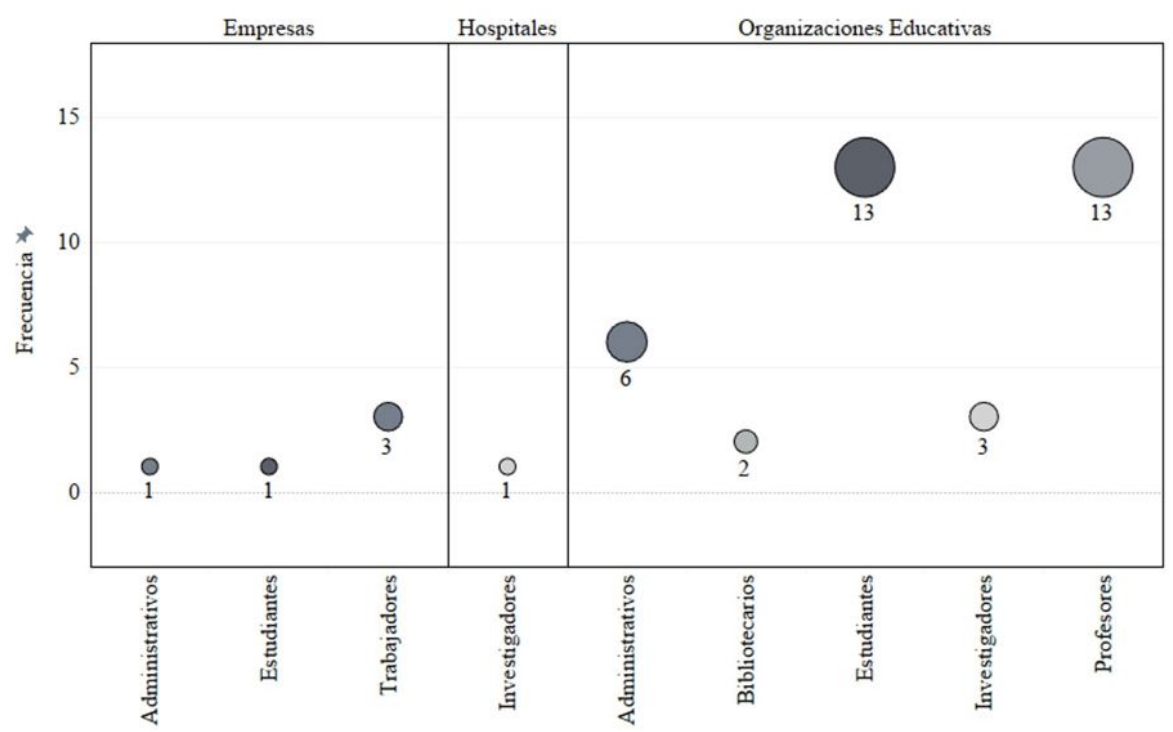

FIGURA 5

Contextos sociales y poblaciones que se han abordado en las investigaciones de la temática Fuente: Elaboración propia

\section{Conceptos relacionados con las estrategias innovadoras}

A través del análisis de contenido efectuado a los resúmenes de las investigaciones se identificaron en las 43 publicaciones los conceptos principales que se manejaron en sus investigaciones (Ver Figura 6). Se encontró que solo cinco de las investigaciones tuvieron como concepto central a las estrategias innovadoras (ver, por ejemplo, las investigaciones: A7, A8, A9, A10 y A13). Los conceptos de Comportamiento Innovador, Emprendimiento y Proceso de Innovación se presentaron con una mediana frecuencia en la literatura (3). Creatividad, Innovación y Tecnologías Computacionales emergieron con una frecuencia menor (2). Y, el resto de los conceptos se identificaron solo en alguna de las publicaciones: Adaptación e Innovación, Administración de la Innovación, Ambiente de Aprendizaje, Ambientes Programados, Aprendizaje en Línea, Competencia Innovadora, Competencias Profesionales, Creación del Conocimiento, E-readiness, Estrategias de intervención, Estudiantes creativos, Innovación Administrativa, Innovación 
Educativa, Innovación para el Aprendizaje, Innovación Tecnológica, Innovaciones Futuras, Innovaciones Organizativas, Liderazgo Educativo, Políticas de Innovación, Prácticas Innovativas, Programas Formativos, Promoción de la Innovación y Sistema de Innovación.

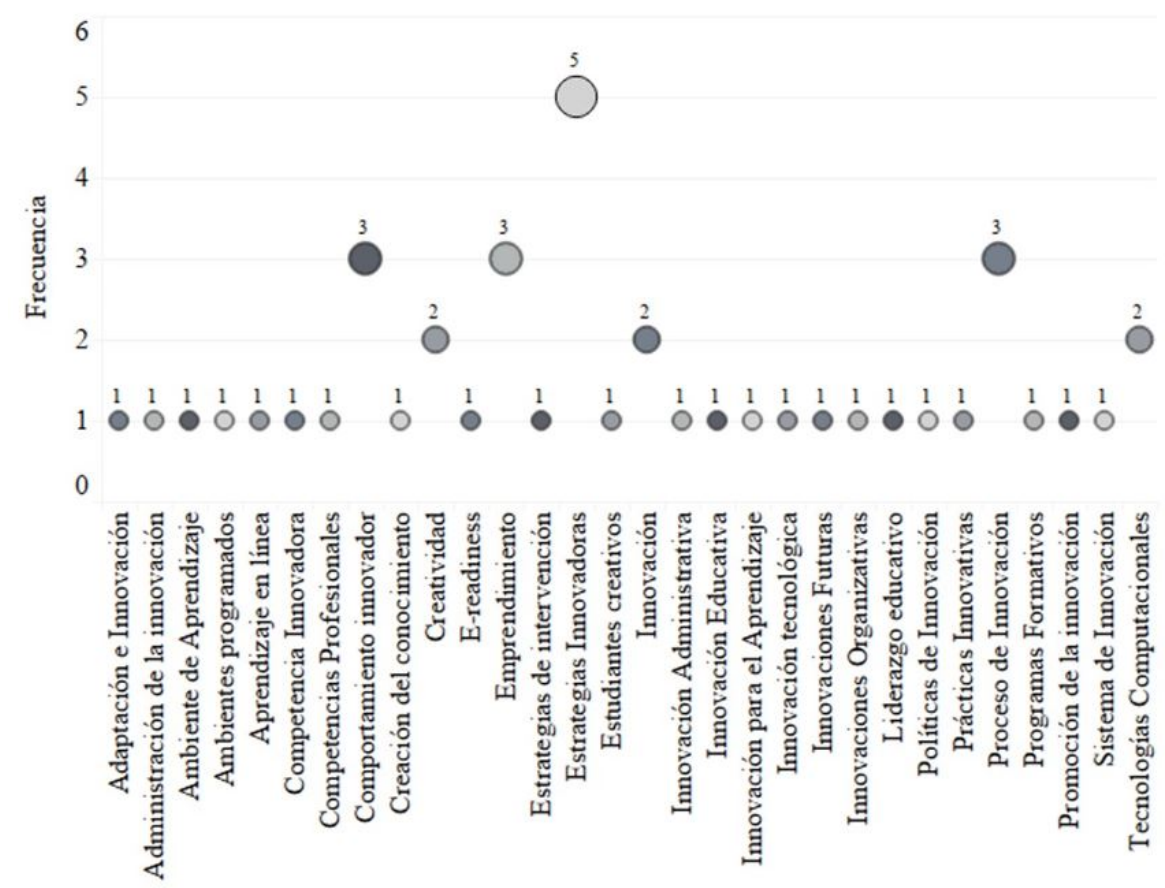

FIGURA 6

Conceptos identificados en las investigaciones sobre estrategias innovadoras Fuente: Elaboración propia

\section{Tipos de estudios e innovación realizados en las investigaciones}

De manera general ubicamos, en la literatura analizada, que la mayor parte de la producción investigativa se concentra en los estudios empíricos (36), le siguen las revisiones sistemáticas de literatura (4) y finalmente los artículos teóricos (3). Dentro de estos tipos de estudios, identificamos el tipo de innovación educativa abordada en las investigaciones (Ver Figura 7) con base en la categorización de la OECD (2016), encontrando que el tipo de innovación correspondiente a las nuevas formas de organizar sus actividades se presenta en la mayoría de las investigaciones (29); véanse las publicaciones A2, A3, A4, A5, A8, A10, A11, A13, A16, A17, A20, A22, A23, A24, A26, A27, A29, A30, A31, A32, A33, A34, A35, A38, A39, A40, A41, A42, A43. Por su parte, la innovación que corresponde a nuevos productos o servicios se identificó en 9 publicaciones (ej. A1, A6, A7, A9, A12, A14, A19, A25, A28). En menor frecuencia (4), se ubicó la innovación de nuevas formas de entregar sus servicios (ej. A15, A18, A36, A37). Y, por último, solo se identificó la investigación A21 en la innovación de nuevas formas de marketing. 


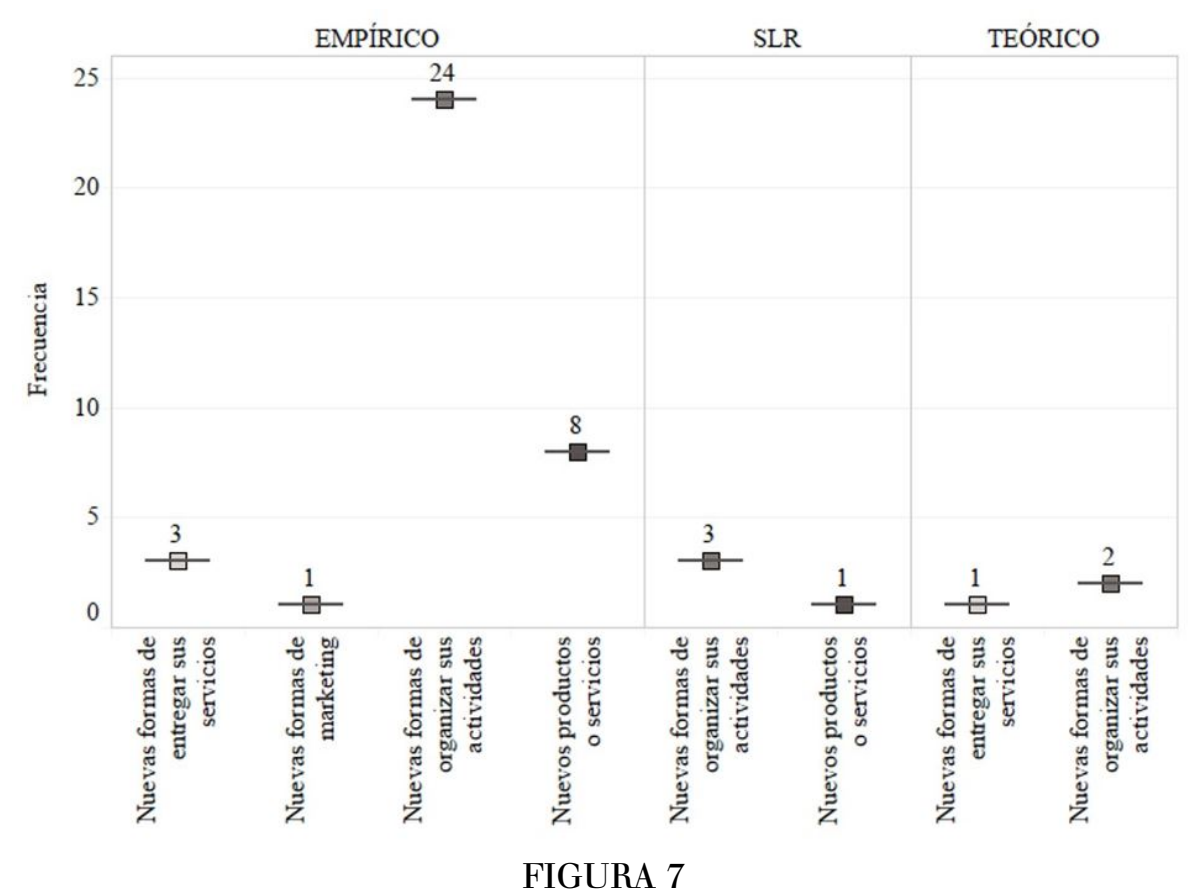

Tipo de innovación y estudios identificados en el mapeo sistemático de literatura Fuente: Elaboración propia

\section{DISCUSIÓN Y CONCLUSIÓN}

Con base en el mapeo de las investigaciones, es posible ubicar una fuerte tendencia en llevar a cabo innovaciones educativas. Las siguientes seis líneas delinean las tendencias en el proceso de innovación educativa y los contextos sociales en los que se está llevando a cabo:

1. Uso de las tecnologías innovadoras. Esta línea se interesa por documentar el impacto de las tecnologías innovadoras en el aprendizaje de los estudiantes (Chipembele y Bwalya, 2016; Kazakoff, Sullivan, y Bers, 2013; Randler, Demirhan, Wüst-Ackermann, y Desch, 2016), estas tecnologías aparecen como un componente mediador importante del proceso innovador, y si bien su incorporación en las organizaciones educativas es incipiente requiere de estrategias pedagógicas planificadas para la consecución de cambios en el proceso educativo, ya que por sí solas no encaminan hacia el progreso y la innovación; es necesario que existan estrategias políticas y organizacionales (Islam y Grönlund, 2016).

2. Obstáculos y retos en la implementación de la innovación educativa. Las investigaciones sobre esta temática se enfocan en los obstáculos y cambios que surgen en los centros educativos para incorporar prácticas innovadoras a través de la experiencia de profesores y practicantes (Fourie y Fourie, 2016), así como las percepciones que tienen las y los profesores sobre la incorporación de recursos tecnológicos en las prácticas educativas (Derbel, 2017; Khanlari, 2016). También se ubican las dificultades que enfrentan los centros educativos para implementar estrategias innovadoras en sus políticas curriculares (Kunc y Likar, 2014) y el papel clave que juega el profesorado en el siglo XXI como agente de cambio en las prácticas innovadoras de los centros educativos (Faulkner, Latham, y Latham, 2016).

3. Agentes educativos en el proceso de innovación. Este grupo de investigaciones explora qué agentes educativos participan en el proceso educativo innovador, por ejemplo, los bibliotecarios (Peng, 2016; Sheih, 2015), estos juegan un papel clave en la implementación de comportamientos 
innovadores en las organizaciones educativas, pues en la estructura de la Universidad facilitan la conexión a estudiantes y docentes con las investigaciones científicas recientes y, a la vez, generan repositorios institucionales que promueven la divulgación del trabajo académico de la universidad expresado en tesis digitales, libros y artículos científicos.

4. El vínculo de las organizaciones educativas con las empresas. Está línea de investigación está cobrando fuerza en países como Singapur. Se dedica a explorar el vínculo de universidades con industrias o empresas, en particular con parques tecnológicos y otros espacios en los que se promueve el emprendimiento educativo y redes de colaboración universidad-empresas (Farré, Sala, y Torres, 2016; Mok y Kan, 2013),

5. Vínculo organizaciones educativas y centros de salud. Emplear las estrategias de innovación educativa en el contexto hospitalario o los centros de salud es otra línea de investigación emergente, en ella se documenta la importancia que tienen las y los investigadores y las universidades para coadyuvar en el desarrollo de los sistemas de innovación (Rey y López, 2017).

6. Impacto de las tecnologías digitales. Finalmente, se establece como una veta importante el impacto de las tecnologías digitales en los centros educativos, dado su importancia como catalizadores de la innovación en el proceso educativo (Kryukov, 2016).

A través de estas tendencias, ubicadas en las investigaciones analizadas, podemos concluir que, bajo las políticas educativas actuales, cada vez más se apertura y flexibiliza el vínculo de la triple hélice (centro educativo-empresa-gobierno) entre las organizaciones educativas con otros contextos de relevancia para el desarrollo económico, político y social de una nación (OECD, 2016). Si bien, el vínculo de las organizaciones educativas con el contexto empresarial y gubernamental, por sí solo, no garantiza que se promuevan estrategias de innovación, sabemos que las universidades son una pieza angular (Piirainen, Andersen y Andersen, 2016; Støren, 2016) para la generación y promoción de la innovación (Mok, 2013), tanto en su papel como un centro formador de las competencias y habilidades indispensables para el proceso innovador, como un puente conector para encontrar nuevas y creativas maneras de combinar el conocimiento externo e interno (Laine, Leino, y Pulkkinen, 2015). Queda con este escrito una invitación a seguir explorando vías que vinculen la innovación con oportunidades de cambio, emprendimiento y mejoramiento de la sociedad en su conjunto.

\section{REFERENCIAS BIBLIOGRÁFICAS}

Aguerrondo, I. (1992). La innovación educativa en América Latina: balance de cuatro décadas. Perspectivas, 22(3), $379-395$.

García, A. (2003). Estrategias para una innovación educativa mediante el empleo de las TIC. Revista Latinoamericana de Tecnología Educativa, 2(1), 33-43.

Hannan, A., English, S. y Silver, H. (1999). Why Innovate? Some Preliminary Findings form a Research Project on "Innovations in Teaching and Learning in Higher Education." Studies in Higher Education, 24(3), 279-289.

Kitchenham, B., Pretorius, R., Budgen, D., Brereton, O. P., Turner, M., Niazi, M. y Linkman, S. (2010). Systematic literature reviews in software engineering - A tertiary study. Information and Software Technology, 52(8), 792 805. doi: https://doi.org/10.1016/j.infsof.2010.03.006

OECD. (2016). Innovating Education and Educating for Innovation: The Power of Digital Technologies and Skills. Paris: OECD Publishing. doi: https://doi.org/http://dx.doi.org/10.1787/9789264265097-en

Ramírez, L. N. y Ramírez, M. S. (2018). El papel de las estrategias innovadoras en educación superior: Retos en las sociedades del conocimiento. Revista Pedagogía, 39(104), 147-170. Recuperado de https://bit.ly/2YA1Gmm

Chipembele, M. y Bwalya, K. (2016). Assessing e-readiness of the Copperbelt University, Zambia: case study. International Journal of Information \& Learning Technology, 33(5), 315-332. doi: https://doi.org/10.1108/IJ ILT-12-2015-0036 
Derbel, F. (2017). Technology-Capable Teachers Transitioning to Technology- Challenged Schools. Electronic Journal of E-Learning, 15(3), 269-280.

Etzkowitz, H. (2012). Triple helix clusters: boundary permeability at university - industry - government interfaces as a regional innovation strategy. Environment and Planning C: Government and Policy, 30(5), 766-779. doi: https://doi.org/10.1068/c1182

Farré, M., Sala, M. y Torres, T. (2016). Network analysis for the study of technological collaboration in spaces for innovation. Science and technology parks and their relationship with the university. International Journal of Educational Technology in Higher Education, 13(8), 1-12. doi: https://doi.org/10.1186/s41239-016-0012-3

Faulkner, J., Latham, G. y Latham, G. (2016). Adventurous Lives: Teacher Qualities for 21st Century Learning. Australian Journal of Teacher Education, 41(4), 137-150. doi: https://doi.org/10.14221/ajte.2016v41n4.9

Fourie, J. E. E. y Fourie, U. U. (2016). Obstacles in Implementing Innovative Practices in Early Childhood Development Centers. Antropologist, 24(3), 799-809.

Frane, A. y Westlund, H. (2013). Innovation in Socio-Cultural Context. New York, N.Y.: Routledge, Editorial.

Islam, M. S. y Grönlund, A. (2016). An international literature review of 1: 1 computing in schools. Journal of Educational Change, 17(2), 191-222. doi: https://doi.org/10.1007/s10833-016-9271-y

Kazakoff, E. R., Sullivan, A. y Bers, M. U. (2013). The Effect of a Classroom-Based Intensive Robotics and Programming Workshop on Sequencing Ability in Early Childhood. Early Chidhood Educational Journal, 41(4), 245-255. doi:https://doi.org/10.1007/s10643-012-0554-5

Khanlari, A. (2016). Teachers' perceptions of the benefits and the challenges of integrating educational robots into primary/elementary curricula. European Journal of Engineering Education, 41(3), 320-330. doi: https://doi.or $\mathrm{g} / 10.1080 / 03043797.2015 .1056106$

Kryukov, V. (2016). Digital Technologies as Education Innovation at Universities. Journal of Internet Banking and Commerce, 21(3), 1-19.

Kunc, P. y Likar, B. (2014). Innovation in secondary education: A threat or a recipe for survival. Didactica Slovenica - Pedagoska Obzorja, 29(2), 32-45.

Laine, K., Leino, M. y Pulkkinen, P. (2015). Open Innovation Between Higher Education and Industry. Journal Knowldege Economy Economy, 6, 589-610. doi: https://doi.org/10.1007/s13132-015-0259-2

Maritz, A., Waal, A. De, Buse, S., Herstatt, C., Lassen, A. y Maclachlan, R. (2014). Innovation education programs: toward a conceptual framework. European Journal of Innovation Management, 17(2), 166-182. doi: https://d oi.org/10.1108/EJIM-06-2013-0051

Mok, K. H. (2013). The quest for an entrepreneurial university in East Asia\#: impact on academics and administrators in higher education. Asian Pacific Educational Review, 14(1), 11-22. doi: https://doi.org/10.1007/s12564-01 3-9249-x

Mok, K. y Kan, Y. (2013). Promoting Entrepreneurship and Innovation in China: Enhancing Research and Transforming University Curriculum. Frontiers of Education in China, 8(2), 173-197. doi: https://doi.org/10 .3868/s110-002-013-0014-3

OECD. (2010). The OECD Innovation Strategy: Getting a Head Start on Tomorrow. Paris: OECD Publishing.

OECD. (2015). The Innovation Imperative: Contributing to Productivity, Growth and Well-Being. Paris: OECD Publishing. doi: https://dx.doi.org/10.1787/9789264239814-en

OECD. (2016). Innovating Education and Educatingfor Innovation: The Power of Digital Technologies and Skills. Paris: OECD Publishing. doi: https://dx.doi.org/10.1787/9789264265097-en

Peng, Y. (2016). A Study of the Relationship among Leader-member Exchange, Creative the University Librarians. Journal of Educational Media and Library Science, 53(1), 27-61. doi: https://doi.org/10.6120/JoEMLS.2016. 531/0044.RS.AM

Petersen, K., Feldt, R., Mujtaba, S. y Mattsson, M. (2007). Systematic Mapping Studies in Software Engineering. EASE'08 Proceedings of the 12th international conference on Evaluation and Assessment in Software Engineering. 
En G. Visaggio, M.T. Baldassarre, S. Linkman y M. Turner, (pp. 68-77). Swindon, UK: BCS Learning \& Development Ltd.

Piirainen, K. A., Andersen, A. D. y Andersen, P. D. (2016). Foresight and the third mission of universities\#: the case for innovation system foresight. Foresight, 18(1), 24-40. doi: https://doi.org/10.1108/FS-04-2014-0026

Ramírez, M.S. (2012). Modelos y estrategias de enseñanza para ambientes innovadores. México: Editorial digital del Tecnológico de Monterrey

Randler, C., Demirhan, E., Wüst-Ackermann, P. y Desch, I. H. (2016). Influence of a Dissection Video Clip on Anxiety, Affect, and Self-Efficacy in Educational Dissection: A Treatment Study. CBE. Life Sciences Education, 15(1), 1-8. doi: https://doi.org/10.1187/cbe.15-07-0144

Rey, J. y López, I. (2017). The fourth mission of hospitals and the role of researchers as innovation drivers in the public healthcare. Revista Española de Documentación Cientifica, 37(1), 1-15. doi: https://doi.org/10.3989/redc.201 4.1 .1062

Richmond, G., y Tatto, M. T. (2016). Innovation in Educational Research. Journal of Teacher Education, 67(5), 360362. doi: https://doi.org/10.1177/0022487116670866

Sheih, C. (2015). Organizational Innovation Behavior in Taiwan's University Libraries: A Quantitative Study.Journal of Educational Media and Library Science, 52(3), 231-267. doi: https://doi.org/10.6120/JoEMLS.2015.523/ 0018.RS.AM

Støren, L. A. (2016). Factors That Promote Innovativeness and Being An Innovative Learner At Work - Results From PIAAC. European Journal of Education, 51(2), 176-192. doi: https://doi.org/10.1111/ejed.12173

Tranfield, D., Denyer, D. y Smart, P. (2003). Towards a Methodology for Developing Evidence-Informed Management Knowledge by Means of Systematic Review. British Journal of Management, 14(3), 207-222. doi: https://doi.org/10.1111/1467-8551.00375

Whitehead, D. (2009). Thoughts on Education and Innovation. Childhood Education, 85(2), 106B.

\section{APÉNDICE A}

\section{Preguntas de Evaluación de la Calidad y Pertinencia de las Investigaciones}

¿El título o resumen contiene un tema relacionado con el área educativa? Sí (1), No (0)

¿Aborda dentro del resumen y palabras clave conceptos relacionados con la innovación educativa? Sí (1), No $(0)$

¿El artículo es de tipo Metodológico (empírico), Teórico o Revisión de Literatura (SLR)? M (1), T (2), $\operatorname{SLR}(3)$

¿En el contenido del resumen se explicita el contexto, escenario, lugar y población en el que se realizó la investigación? Sí (1), No (0)

\section{APÉNDICE B}

\section{- Contexto: Organizaciones educativas}

A1. Fourie, J. E. y Fourie, U. U. (2016). Obstacles in Implementing Innovative Practices in Early Childhood Development Centers. Anthropologist, 24(3), 799-809.

A2. Titrek, O. (2015). The Level of Innovation Management of School Principals' in Turkey. Anthropologist, 19(2), 449-456 
A4. Eguizábal, A. J., Cámara, C. P., Val, P. A., Palmero, A. J., Rico, I. L., de la Torre Cruz, T. y Adánez, Á. G. ¿Cómo organizar un congreso científico de calidad? Claves y variables del éxito del XV Congreso Nacional Iberoamericano de Pedagogía. Bordón. Revista de Pedagogía, 65(3), 39-55. http://doi:10.13042/23162

A6. Kristiansson, M. R. y Jochumsen, H. (2015). How to implement entrepreneurship in LIS education: a Danish example. BiD: textos universitaris de biblioteconomia i documentació, $35 \mathrm{http}: / / \mathrm{bid} . u b . e d u / e n / 35$ /kristiansson.htm

A7. Randler, C., Demirhan, E., Wüst-Ackermann, P. y Desch, I. H. (2016). Influence of a Dissection Video Clip on Anxiety, Affect, and Self-Efficacy in Educational Dissection: A Treatment Study. CBE Life Sciences Education, 15(1), ar1. http://doi.org/10.1187/cbe.15-07-0144

A9. Fernández-Díaz, E., Calvo, A. y Rodríguez-Hoyos, C. (2014). Towards a collaborative action research in Spain to improve teaching practice. Educational Action Research, 22(3), 397-410. http://dx.doi.org/10. 1080/09650792.2014.882261

A16. Mok, K. H. y Yue, K. A. N. (2013). Promoting Entrepreneurship and Innovation in China: Enhancing Research and Transforming University Curriculum. Frontiers of Education in China, 8(2), 173-197. https://doi.org/10.3868/s110-002-013-0014-3

A17. Kowang, T. O., Long, C. S. y Rasli, A. (2015). Innovation management and performance framework for research university in Malaysia. International Education Studies, 8(6), 32-35. http://dx.doi.org/10.553 9/ies.v8n6p32

A19. Chen, B., Scardamalia, M. y Bereiter, C. (2015). Advancing knowledge\#building discourse through judgments of promising ideas. International Journal of Computer-Supported Collaborative Learning, 10(4), 345-366. doi: https://doi.org/10.1007/s11412-015-9225-z

A20. Durkin, M., Howcroft, B. y Fairless, C. (2016). Product development in higher education marketing. International Journal of Educational Management, 30(3), 354-369. https://doi.org/10.1108/IJEM-11-201 4-0150

A22. Taajamaa, V., Eskandari, M., Karanian, B., Airola, A., Pahikkala, T. y Salakoski, T. (2016). O-CDIO: Emphasizing Design Thinking in CDIO Engineering Cycle. International Journal of Engineering Education, 32(3b), 1530-1539.

A25. McHardy, P. y Vershinina, N. A. (2014). The role of autocatalysis in learner's networks. The International Journal of Management Education, 12(3), 271-282. https://doi.org/10.1016/j.ijme.2014.09. 001

A26. Cerinsek, G., Hribar, T., Glodez, N. y Dolinsek, S. (2013). Which are my future career priorities and what influenced my choice of studying science, technology, engineering or mathematics? Some insights on educational choice - case of Slovenia. International Journal of Science Education, 35(17), 2999-3025. ht tp://dx.doi.org/10.1080/09500693.2012.681813

A29. Peng, Y. P. (2013). A Study of the Relationship among Leader-Member Exchange, Job Autonomy and Organizational Citizenship Behavior of the University Librarians in Taiwan. Journal of Educational Media \& Library Sciences, 50(4), 491-525. doi: https://doi.org/10.6120/JoEMLS.2013.504/0557.RS.AM

A30. Sheih, C.S. (2015). Organizational innovation behavior in Taiwan's university libraries: A quantitative study. Journal of Educational Media and Library Science, 52(3), art. no. 2, 231-267. doi: https ://doi.org/10.6120/JoEMLS.2015.523/0018.RS.AM

A31. Nielsen, J. A. (2015). Assessment of innovation competency: A thematic analysis of upper secondary school teachers' talk. The Journal of Educational Research, 108(4), 318-330. http://dx.doi.org/10.1080/00 220671.2014 .886178

A32. Jablokow, K. W., DeFranco, J. F., Richmond, S. S., Piovoso, M. J. y Bilén, S. G. (2015). Cognitive style and concept mapping performance. Journal of Engineering Education, 104(3), 303-325. Doi: https:// doi.org/10.1002/jee.20076 
A8. Petko, D., Egger, N., Cantieni, A. y Wespi, B. (2015). Digital media adoption in schools: Bottomup, top-down, complementary or optional? Computers \& Education, 84, 49-61. https://doi.org/10.1016/j .compedu.2014.12.019

A11. Bocconi, S., Kampylis, P. y Punie, Y. (2013). Framing ICT\#enabled Innovation for Learning: the case of one\#to\#one learning initiatives in Europe. European Journal of Education, 48(1), 113-130. doi: htt ps://doi.org/10.1111/ejed.12021

A12. Khanlari, A. (2016). Teachers' perceptions of the benefits and the challenges of integrating educational robots into primary/elementary curricula. European Journal of Engineering Education, 41(3), 320-330. http://dx.doi.org/10.1080/03043797.2015.1056106

A13. Kunc, P. y Likar, B. (2014). Innovation in secondary education: A threat or a recipe for survival. [Inovativnost v srednjem šolstvu - Grožnja ali recept za preživetje] Didactica Slovenica - Pedagoska Obzorja, 29(2), 32-45.

A14. Kazakoff, E. R., Sullivan, A. y Bers, M. U. (2013). The effect of a classroom-based intensive robotics and programming workshop on sequencing ability in early childhood. Early Childhood Education Journal, 41(4), 245-255. doi:https://doi.org/10.1007/s10643-012-0554-5.

A15. Derbel, F. (2017). Technology-Capable Teachers Transitioning to Technology-Challenged Schools. Electronic Journal of e-Learning, 15(3), 269-280.

A18. Ng, W. S. T. y Wilson, E. (2017). A perspective of teachers' appropriation of educational innovations. International Journal for Lesson \& Learning Studies, 6(3), 202-215. https://doi.org/10.1108/ IJLLS-12-2016-0052

A28. Islam, M. S. y Grönlund, Å. (2016). An international literature review of 1: 1 computing in schools. Journal of Educational Change, 17(2), 191-222. doi: https://doi.org/10.1007/s10833-016-9271-y

A33. Fazlagić, J. y Erkol, A. (2015). Knowledge mobilisation in the Polish education system. Journal of Education for Teaching, 41(5), 541-554. http://dx.doi.org/10.1080/02607476.2015.1105539

A36. Baturay, M. H., Gökçearslan, Ş. y Şahin, Ş. (2017). Associations among Teachers' Attitudes towards Computer-Assisted Education and TPACK Competencies. Informatics in Education, 16(1), 1-23. Doi: ht tps://doi.org/10.15388/infedu.2017.01

A37. Chipembele, M., Chipembele, M., Bwalya, K. J., y Bwalya, K. J. (2016). Assessing e-readiness of the Copperbelt University, Zambia: case study. The International Journal of Information and Learning Technology, 33(5), 315-332. doi: https://doi.org/10.1108/IJILT-12-2015-0036

A40. Faulkner, J. y Latham, G. (2016). Adventurous lives: Teacher qualities for 21st century learning. Australian Journal of Teacher Education (Online), 41(4), 137. http://dx.doi.org/10.14221/ajte.2016v41n4 .9

A41. Goldman, S. R. y Scardamalia, M. (2013). Managing, understanding, applying, and creating knowledge in the information age: Next-generation challenges and opportunities. Cognition and Instruction, 31(2), 255-269. http://dx.doi.org/10.1080/10824669.2013.773217

A43. Wang, R., De Donder, L., De Backer, F., Shihua, L., Honghui, P., Thomas, V. y Lombaerts, K. (2016). Back to school in later life: Older Chinese adults' perspectives on learning participation barriers. Educational Gerontology, 42(9), 646-659. http://dx.doi.org/10.1080/03601277.2016.1205385

A38. Hayes, H. H. (2014). The scholarship of application in the context of academic entrepreneurialism: A review of the discursive field. Asian Education and Development Studies, 3(3), 292-302. https://doi.org/ 10.1108/AEDS-08-2014-0038

A39. Mok, K. H. (2013). The quest for an entrepreneurial university in East Asia: impact on academics and administrators in higher education. Asia Pacific Education Review, 14(1), 11-22. https://doi.org/10.10 07/s12564-013-9249-x 
A3. Tan, O. K., Choi, S. L., Rasli, A. y Goh, C. F. (2015). Perception versus Performance Indicators: A Study of Innovation Performance in a Research University. Asian Social Science, 12(1), 24-29. http://doi: $10.5539 /$ ass.v $12 \mathrm{n} 1 \mathrm{p} 24$

A24. Camilleri, M. A. y Camilleri, A. (2016). Education and social cohesion for economic growth. International Journal of Leadership in Education, 19(5), 617-631. http://dx.doi.org/10.1080/13603124.20 14.995721

A27. Groener, Z. (2013). Skills development and structural change: Possibilities for and limitations of redressing structural racial inequalities in South Africa. International Review of Education, 59(6), 723-749.

A5. Sellar, S. y Lingard, B. (2014). The OECD and the expansion of PISA: New global modes of governance in education. British Educational Research Journal, 40(6), 917-936. http://doi:10.1002/berj.3 120

\section{. Contexto: Empresarial}

A10. Støren, L. (2016). Factors That Promote Innovativeness and Being An Innovative Learner At WorkResults From PIAAC. European Journal of Education, 51(2). doi: https://doi.org/10.1111/ejed.12173

A21. Farré-Perdiguer, M., Sala-Rios, M. y Torres-Solé, T. (2016). Network analysis for the study of technological collaboration in spaces for innovation. Science and technology parks and their relationship with the university. International Journal of Educational Technology in Higher Education, 13(1), 1-12. doi: https://doi.org/10.1186/s41239-016-0012-3

A23. Walczak, M., Uziak, J., Oladiran, M. T., Baeza, C. C. y Paez, P. T. (2013). Industry Expectations of Mechanical Engineering Graduates. A Case Study in Chile. International Journal of Engineering Education, 29(1), 181-192.

A34. Shanker, R., Bhanugopan, R., Van der Heijden, B. I. y Farrell, M. (2017). Organizational climate for innovation and organizational performance: The mediating effect of innovative work behavior. Journal of Vocational Behavior, 100, 67-77. https://doi.org/10.1016/j.jvb.2017.02.004

A35. Nikolova, I., Van Ruysseveldt, J., De Witte, H. y Van Dam, K. (2014). Learning climate scale: Construction, reliability and initial validity evidence. Journal of Vocational Behavior, 85(3), 258-265. https ://doi.org/10.1016/j.jvb.2014.07.007

\section{- Contexto: Hospitales}

A42. Rey-Rocha, J. y López-Navarro, I. (2014). The fourth mission of hospitals and the role of researchers as innovation drivers in the public healthcare sector. Revista Española de Documentación Científica, 37(1), 1-15. Doi: https://doi.org/10.3989/redc.2014.1.1062

\section{BY-NC-ND}

